\title{
ÓLEO REFINADO DE SOJA PARA ACIONAMENTO DE MOTORES DIESEL
}

Haroldo Carlos Fernandes ${ }^{1}$, Guilherme Ladeira dos Santos², Wagner da Cunha Siqueira ${ }^{3}$, Lara Santana Fernandes ${ }^{4}$, Flavio Coutinho Longui ${ }^{5}$

\section{RESUMO}

O objetivo do trabalho foi verificar a viabilidade da utilização do Óleo Refinado de Soja (ORS) em motores de ciclo diesel de baixa potência. O trabalho foi realizado no Laboratorio de Mecanização Agricola da Universidade Federal de Viçosa. Para a representação das curvas de rendimento do motor, os dados foram tabulados e analisados estatisticamente com o auxílio do software SAS e as equações geradas foram testadas pelo teste $\mathrm{T}$ e $\mathrm{F}$, e para a representação das curvas de rendimento foi utilizado analises de regressão. As características analisadas foram: a viscosidade cinemática das misturas em diferentes proporções de ORS e óleo diesel com um viscosímetro Saybolt Universal; os rendimentos foram realizados em um motor Yanmar modelo NSB75, com potência nominal de $5.5 \mathrm{cv}$ a $1800 \mathrm{rpm}$, monocilíndrico e injeção direta, sendo o combustível utilizado para os ensaios as misturas de ORS e óleo diesel nas proporções de $0,2,10,20,30$, 40, e 50\%. Verificou-se que o aquecimento é uma técnica viável para a redução da viscosidade e que para atender Portaria ANP 310, as proporções têm que ser pequenas e deverão ser aquecidas, e o aquecimento é mais eficiente em proporções maiores de ORS. Foi verificado aumento da potência e do torque em função do aumento da proporção de ORS, com relação ao consumo específico não foram encontras diferenças significativas. Em relação ao custo concluiu-se que em $\mathrm{R} \mathrm{kW}^{-1} \mathrm{~h}^{-1}$, não ocorreram diferenças significativas. Conclui-se que, a utilização do óleo vegetal se mostrou viável, de forma técnica e outros trabalhos deverão ser realizados para avaliar o motor em condições de trabalho por longo período, para avaliar as condições de desgaste e de manutenção.

Palavras-chave: Biocombustível, motor diesel e óleo vegetal.

\section{ABSTRACT}

\section{USE OF REFINED SOYBEAN OIL IN DIESEL ENGINES LOW POWER}

The objective of this study was to assess the feasibility of using the Refined Soybean Oil (SAB) in diesel engines of low power. The work was conducted in Laboratory of Agricultural Mechanization of the Federal University of Viçosa. For the representation of the yield curves of the engine, the data were statistically analyzed with SAS software and the equations generated were tested using $\mathrm{T}$ and $\mathrm{F}$, and the depiction of the yield curves was used for regression analysis. The characteristics examined were: kinematic viscosity of the mixtures in different proportions of SAB and diesel oil with a Saybolt Universal viscometer; yields were performed on a model NSB75 Yanmar engine, with rated power of 5.5 bhp at $1800 \mathrm{rpm}$, and cylinder direct injection, and the fuel used for testing mixtures of ORS and diesel in the proportions of 0,2 , $10,20,30,40$, and $50 \%$. It was found that heating is a viable technique for reducing the viscosity and to meet ANP 310 , the proportions have to be small and should be heated, and heating is more efficient in larger proportions of SAB. It was found increased power and torque due to the increase in the proportion of $\mathrm{SAB}$, with respect to the specific consumption were not found significant differences. For the cost it was concluded that for $\$ \mathrm{~kW}^{-1} \mathrm{~h}^{-1}$, there were no significant differences. We conclude that the use of vegetable oil to be viable, so technical and other studies should be conducted to assess the engine working conditions for long periods, to assess the conditions of wear and maintenance

Keywords: biofuels, diesel and vegetable oil.

\section{Recebido para publicação em 21/09/2009. Aprovado em 16/03/2010}

1- Engenheiro Agrícola, Dr, Professor Associado, Universidade Federal de Viçosa - MG - haroldo@ufv.br

2- Engenheiro Florestal, Universidade Federal de Viçosa -MG -santosladeira@hotmail.com.

3- Mestrando em Mecanização Agrícola, Universidade Federal de Viçosa - MG - wagnercsiqueira@yahoo.com.br.

4- Graduanda em Engenharia de Alimentos, Universidade Federal de Viçosa - MG - lara.fernandes@ufv.br .

5- Mestrando em Mecanização Agrícola, Universidade Federal de Viçosa - MG - flavio.longui@ufv.br. 


\section{INTRODUÇÃO}

O Brasil produz $86 \%$ da energia nele consumida, sendo o restante, $14 \%$, importado na forma de petróleo e seus derivados e de energia elétrica. Cerca de $41 \%$ da Oferta Interna de Energia (OIE) no Brasil tem origem em fontes renováveis, enquanto no mundo a taxa é de $14 \%$ e, nos países desenvolvidos, de apenas $6 \%$. De toda a energia renovável produzida, a biomassa responde por $27 \%$, e a energia hidráulica por 14\% (MINISTÉRIO DE MINAS E ENERGIA, 2004).

A constante incógnita relacionada ao futuro dos combustíveis derivados de petróleo e a necessidade sempre presente de redução das emissões de contaminantes atmosféricos faz com que a substituição do óleo diesel convencional e da gasolina seja orientada, em diferentes locais, pela busca de alternativas oriundas da biomassa. $\mathrm{O}$ ramo da agricultura que objetiva a produção e o aproveitamento da biomassa para fins energéticos denomina-se "agroenergética" (GONZÁLEZ, 1994). Segundo Delgado (1994) a utilização da biomassa é a alternativa mais viável, pois o seu importante produto - a energia - é o único com demanda ilimitada. Potencializados como a uma das melhores alternativa ao óleo diesel em motores de ignição por compressão, os óleos vegetais podem ser usados in natura, ou esterificados (biodiesel), ou ainda em misturas com o óleo diesel convencional (DELGADO, 1994).

O Brasil consome cerca de 34 milhões de $\mathrm{m}^{3}$ ano-1 de óleo diesel, dos quais quase 30 milhões são destinados ao setor de transporte;, e em média são importados cerca de 6 milhões de $\mathrm{m}^{3}$ ano ${ }^{-1}$ de diesel, a um custo próximo dos US $\$ 1,22$ bilhão; por isso fazse necessário o aproveitamento de óleos vegetais para acionar veículos (SANT'ANNA, 2003). Este fato não é recente, pois Rudolf Diesel inventor do motor a diesel, propôs a utilização de óleo de amendoim como combustível há cerca de cem anos, porém, o baixo custo do petróleo inibiu o projeto.

O Brasil apresenta vantagens em relação aos países do primeiro mundo, tendo em vista as grandes áreas agricultáveis, as diversas espécies que podem ser cultivadas ou exploradas de forma sustentável, além das características climáticas que privilegiam o país (INOUE, 2008).

Em termos de área, o Brasil explora menos de um terço de sua área agricultável, o que constitui a maior fronteira para expansão agrícola do mundo. O potencial é de cerca de 150 milhões de hectares, sendo 90 milhões referentes às novas fronteiras, e os outros 60 referentes às terras de pastagens que podem ser convertidas em exploração agrícola em curto prazo (IICA, 2007).

O óleo vegetal misturado ao óleo diesel na forma in natura na substituição do óleo diesel são alternativas viáveis, considerando especialmente os aspectos sociais, ambientais e econômicos. Em contrapartida o óleo vegetal pode ser utilizado in natura, sendo extraído do grão ou amêndoa através de processos físicos (MORAES, 1980).

De acordo com Fender (2009) a utilização do óleo vegetal na forma in natura poderá ter vantagens em relação ao biodiesel apresentando possibilidade de total independência dos derivados de petróleo; não requer investimentos em longo prazo, em especial, com equipamentos e apresenta imediato retorno do capital empregado e incentivo à produção de sementes oleaginosas.

De acordo com o trabalho realizado por Inoue (2009), não ocorreu formação de resíduos na câmara de combustão que necessitasse de descarbonização antes de 300 horas de operação do motor, porém quando o óleo vegetal misturado ao diesel, o intervalo de troca dos lubrificantes deve ser reduzido devido a sua contaminação. Para este mesmo autor a utilização de óleo vegetal é uma alternativa viável em comunidades distantes de centros de distribuição de combustível.

Objetivou-se com esse trabalho verificar a viabilidade da utilização do óleo refinado de soja em motores diesel de baixa potência.

\section{MATERIAL E MÉTODOS}

As avaliações foram conduzidas no Laboratório de Mecanização Agrícola do Departamento de Engenharia Agrícola LMA/ DEA da Universidade Federal de Viçosa (UFV). Para a representação das curvas de rendimento do motor, os dados foram tabulados e analisados estatisticamente com o auxílio do software SAS e as equações geradas foram testadas pelo teste $\mathrm{T}$ e $\mathrm{F}$, e para a representação das curvas de rendimento foi utilizado analises de regressão. As características analisadas foram: a viscosidade cinemática das misturas em diferentes proporções de Óleo Refinado de Soja (ORS) adquirido em supermercado e óleo diesel e o rendimento do motor a diesel com as misturas em estudo. 
Determinação das viscosidades cinemáticas das misturas de ORS e óleo diesel.

Para determinação da viscosidade foi utilizado um Viscosímetro Saybolt instalado no Laboratório de Mecanização Agrícola, onde as avaliações foram realizadas com base na norma NBR 10441 (2007). Foram analisadas as viscosidades cinemáticas das amostras de diesel (OD), e óleo vegetal (OV) de $\mathrm{OV}_{10}, \mathrm{OV}_{30}, \mathrm{OV}_{50}, \mathrm{OV}_{70}$, e (ORS) puro para as temperaturas de 40,60 e $80^{\circ} \mathrm{C}$. O tempo de coleta da amostra no recipiente padrão de $60 \mathrm{ml}$, que é dado em Segundo Saybolt Universal (SSU), foi convertido para a unidade em centistroke (cSt) com o auxílio do ábaco apresentado por Barguer et al. (1966).

\section{Determinação do rendimento do motor}

As avaliações de rendimento foram realizadas em um motor Yanmar modelo NSB 75, com potência nominal de $5.5 \mathrm{cv}$ a $1800 \mathrm{rpm}$, monocilíndrico e de injeção direta, o combustível utilizado para os ensaios são as misturas de Óleo Refinado de Soja (ORS) e óleo diesel nas proporções de $0,2,10,20$, 30,40 , e $50 \%$ de ORS no OD.

Para a realização das avaliações foi utilizado um dinamômetro de rotação do tipo transmissão montado em berço, fluxômetro de combustível, tacômetro digital e cronômetro. As avaliações tiveram como referência a Norma NBR ISO 1585 (1996), onde foi utilizado um termômetro de bulbo úmido e seco e dados de pressão barométrica da estação meteorológica da UFV.

Foi utilizado o tempo de coleta de 5 minutos, coletando-se a rotação, carga no dinamômetro, consumo e temperaturas, foram realizadas 3 (três) repetições para cada combustível, de forma aleatória. Após a realização das avaliações foi coletada a rotação do motor sem carga, para verificar a regulagem do motor, que foi fixada para o óleo diesel em $2440 \mathrm{rpm}$.

As cargas aplicadas pelo dinamômetro no motor foram de $7 ; 9 ; 11 ; 13 ; 15 ; 17 ; 19$ e 21,5 lb. O motor foi acoplado ao dinamômetro com o auxílio de polias e correias do tipo $\mathrm{V}$, com relação de transmissão de 1:1.9.

\section{Análise econômica da substituição do óleo diesel pela mistura do combustível}

Para o cálculo do custo de substituição do óleo diesel pelas misturas com óleo vegetal levou-se em consideração o custo de aquisição do $\mathrm{OD}, \mathrm{R} \$ 1,90$, assim como o B2 (proporção de biodiesel no diesel em porcentagem), que também foi adquirido ao custo de R\$1,90. O ORS foi comprado ao preço de $\mathrm{R} \$ 1,80$. Levou-se em consideração para os cálculos, os valores da densidade dos combustíveis testados, além do consumo específico e potência na rotação de trabalho estipulado.

\section{Análise dos dados}

Após a coleta, os dados de viscosidade e de rendimento do motor, foram tabulados e analisados estatisticamente com o auxílio do software SAS e as equações geradas foram testadas pelo teste $\mathrm{T}$ e $\mathrm{F}$.

\section{RESULTADOS E DISCUSSÃO}

\section{Viscosidade Cinemática}

Na Figura 1 são apresentados os valores de viscosidade do Óleo Refinado de Soja (ORS) para diversas temperaturas e proporção de ORS na mistura do combustível, que para uma condição de temperatura $40{ }^{\circ} \mathrm{C}$, nenhuma das proporções de Óleo Vegetal (OV) atendeu a Portaria ANP 310, fato que pode ser explicado já que o óleo diesel possui sua viscosidade cinemática perto do limite superior estabelecido pela portaria. No caso do combustível aquecido até $60{ }^{\circ} \mathrm{C}$, verificou-se que uma mistura de até $4 \%$ se enquadra na Portaria da ANP, e quando a temperatura foi elevada para $80^{\circ} \mathrm{C}$, verificou-se que a mistura pode conter até uma proporção de $17 \%$ de ORS.

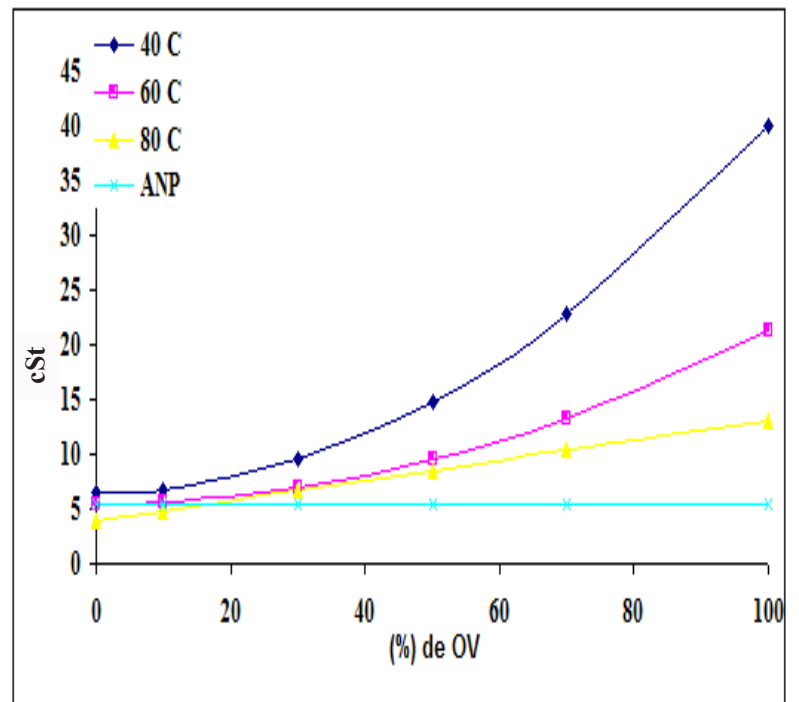

Figura 1.Curvas da Viscosidade Cinemática em função da porcentagem de óleo vegetal 
O simples aquecimento da mistura de $40^{\circ}$ para $80{ }^{\circ} \mathrm{C}$ reduziu em $65 \%$ a viscosidade para uma condição de ORS puro, porém é na faixa de 40 a $60{ }^{\circ} \mathrm{C}$ que ocorre a maior influência da temperatura na redução da viscosidade. Nas curvas apresentadas (Figura 1), pode se verificar ainda que quanto maior a proporção de ORS, maior a influência da temperatura na redução da viscosidade.

Resultado semelhante foi encontrado por Siqueira (2010) na utilização de misturas de óleo de soja reutilizado com óleo diesel em um trator agrícola de pneus onde verificou-se que o aquecimento do $100 \%$ OSR de 40 para $90{ }^{\circ} \mathrm{C}$ reduziu em até $90,4 \%$ a sua viscosidade.

Castro (1999) estudando a viscosidade do óleo de babaçu verificou que, a viscosidade decresceu consideravelmente em presença a altas temperaturas.

\section{Rendimento do motor}

Foram geradas equações para as curvas de potência, torque e consumo específico que são apresentadas nos Quadros 1, 2 e 3 respectivamente.

A potência na rotação de trabalho foi de 4,79 , 5,10 e $5,08 \mathrm{~kW}$, respectivamente para o OD, B2 e OV02. Verifica-se que variações em relação ao OD foram de $6,46 \%$ a mais de potência a mais para o B2 e $6,10 \%$ a mais de potência para o OV02, esta condição pode ser verificada na Figura 2.

Observa-se que o torque na rotação de $2350 \mathrm{rpm}$, apresentou valores de $19,35,20,76$ e $20,77 \mathrm{mN}$, respectivamente para o OD, B2 e OV02. Verifica-se uma pequena variação em relação ao OD nos valores de $7,29 \%$ a mais para o $\mathrm{B} 2$ e $7,31 \%$ a mais para o OV02, como pode ser observado na Figura 3.

No consumo específico observa-se que para a rotação de $2350 \mathrm{rpm}$, foram encontradas os valores de 257,262 e $240 \mathrm{gkW} \mathrm{h}^{-1}$ respectivamente para o $\mathrm{OD}, \mathrm{B} 2$ e OV02. As variações em relação ao OD foram de $1,98 \%$ a mais para o B2 e de $6,40 \%$ a menos para o OV02, como observado na Figura 4.

Quadro 1. Equações geradas para estimar a potência

\begin{tabular}{lcc}
\hline \multicolumn{1}{c}{ Equação } & $\mathbf{R}^{2}$ & Combust. \\
\hline$N=-0,00008812 n^{2}+0,36684 n-370,6263$ & 99,68 & OD \\
\hline$N=-0,00014714 n^{2}+0,64466 n-697,2562$ & 99,70 & $\mathrm{~B}_{2}$ \\
\hline$N=-0,05198 n-127,25145$ & 98,75 & $\mathrm{OV}_{02}$ \\
\hline
\end{tabular}

em que,

$\mathrm{N}$ - Potência do Motor em kW;

$n$ - Rotação do Motor em rpm.

Quadro 2. Equações geradas para estimar o torque

\begin{tabular}{lcc}
\hline \multicolumn{1}{c}{ Equação } & $\mathbf{R}^{2}$ & Combust. \\
\hline$\tau=-0,0002583 n^{2}+1,01214 n-932,65318$ & 99,71 & OD \\
\hline$\tau=-0,00050852 n^{2}+2,18969 n-2316,64543$ & 99,70 & $\mathrm{~B}_{2}$ \\
\hline$\tau=-0,22063 n+539,31574$ & 99,03 & $\mathrm{OV}_{02}$ \\
\hline
\end{tabular}

em que,

$\tau$ - Torque do Motor em Nm;

$n$ - Rotação do Motor em rpm.

Quadro 3. Equações geradas para estimar o consumo específico

\begin{tabular}{ccc}
\hline \multicolumn{1}{c}{ Equação } & R $^{2}$ & Combust. \\
\hline$q=0,02335 n^{2}-109,24764 n+128038$ & 99,12 & OD \\
\hline$q=0,02737 n^{2}-128,35202 n+150738$ & 93,34 & B2 \\
\hline$q=0,03184 n^{2}-149,426092 n+175555$ & 99,03 & OV $_{02}$ \\
\hline
\end{tabular}

em que,

$q$ - Consumo Específico em $\mathrm{g} \mathrm{kW}^{-1} \mathrm{~h}^{-1}$;

$n$ - Rotação do Motor em rpm. 
Quadro 4. Equações do desempenho do motor para as diferentes misturas de combustível

\begin{tabular}{|c|c|c|c|}
\hline & & Equações & $\mathrm{R}^{2}$ \\
\hline \multirow{3}{*}{ OD } & Potência & $N=-0,00008812 n^{2}+0,36684 n-370,6263$ & 99,68 \\
\hline & Torque & $\tau=-0,0002583 n^{2}+1,01214 n-932,65318$ & 99,71 \\
\hline & Consumo & $q=0,02335 n^{2}-109,24764 n+128038$ & 99,12 \\
\hline \multirow{3}{*}{ B2 } & Potência & $N=-0,00014714 n^{2}+0,64466 n-697,2562$ & 99,70 \\
\hline & Torque & $\tau=-0,00050852 n^{2}+2,18969 n-2316,64543$ & 99,70 \\
\hline & Consumo & $q=0,02737 n^{2}-128,35202 n+150738$ & 93,34 \\
\hline & & Equações & $\mathrm{R}^{2}$ \\
\hline \multirow{3}{*}{ OV02 } & Potência & $N=-0,05198 n-127,25145$ & 98,75 \\
\hline & Torque & $\tau=-0,22063 n+539,31574$ & 99,03 \\
\hline & Consumo & $q=0,03184 n^{2}-149,426092 n+175555$ & 96,46 \\
\hline \multirow{3}{*}{ OV10 } & Potência & $N=-0,00017773 n^{2}+0,78782 n-867,81123$ & 99,38 \\
\hline & Torque & $\tau=-0,00063165 n^{2}+2,76571 n-2990,66116$ & 99,41 \\
\hline & Consumo & $q=0,0231 n^{2}-108,12083 n+126795$ & 96,22 \\
\hline \multirow{3}{*}{ OV20 } & Potência & $N=-0,04676 n-115,12714$ & 98,50 \\
\hline & Torque & $\tau=-0,1984 n+487,69712$ & 98,66 \\
\hline & Consumo & $q=0,02335 n^{2}-109,92883 n+129655$ & 99,36 \\
\hline \multirow{3}{*}{ OV30 } & Potência & $N=-0,00017875 n^{2}+0,79728 n-880,99707$ & 98,87 \\
\hline & Torque & $\tau=-0,19572 n+481,62457$ & 97,63 \\
\hline & Consumo & $q=0,0248 n^{2}-116,6888 n+137524$ & 98,00 \\
\hline \multirow{3}{*}{ OV40 } & Potência & $N=-0,00015496 n^{2}+0,68528 n-748,87582$ & 99,14 \\
\hline & Torque & $\tau=-0,20197 n+497,65322$ & 98,31 \\
\hline & Consumo & $q=0,02606 n^{2}-122,87975 n+145127$ & 95,62 \\
\hline \multirow{3}{*}{ OV50 } & Potência & $N=-0,00018421 n^{2}+0,8255 n-916,51286$ & 99,78 \\
\hline & Torque & $\tau=-0,00065278 n^{2}+2,89187 n-3165,93454$ & 99,78 \\
\hline & Consumo & $q=0,0275 n^{2}-130,04433 n+154016$ & 95,04 \\
\hline
\end{tabular}

em que,

$\mathrm{N}$ - Potência do Motor em kW;

$\tau$ - Torque do Motor em Nm;

$q$ - Consumo Específico em $\mathrm{g} \mathrm{kW}^{-1} \mathrm{~h}^{-1}$;

$n$ - Rotação do Motor em rpm.

Quadro 5. Equações gerais para estimativa do desempenho do motor para todos os combustíveis avaliados.

\begin{tabular}{cccc}
\hline \multirow{3}{*}{ Geral } & Potência & $N=-0,00011412 n^{2}+0,49096 n-0,00011412 \rho-518,65452$ & 98,28 \\
\cline { 2 - 4 } & Torque & $\tau=-0,00038298 n^{2}+1,60503 n-9,46898 \rho-1636,96545$ & 98,41 \\
\cline { 2 - 4 } & Consumo & $q=0,02046 n^{2}-96,07405 n+113057$ & 82,41 \\
\hline
\end{tabular}

em que,

$\mathrm{N}$ - Potência do Motor em kW;

$\tau$ - Torque do Motor em Nm;

$q$ - Consumo Específico em $\mathrm{g} \mathrm{kW}^{-1} \mathrm{~h}^{-1}$;

$n$ - Rotação do Motor em rpm. 


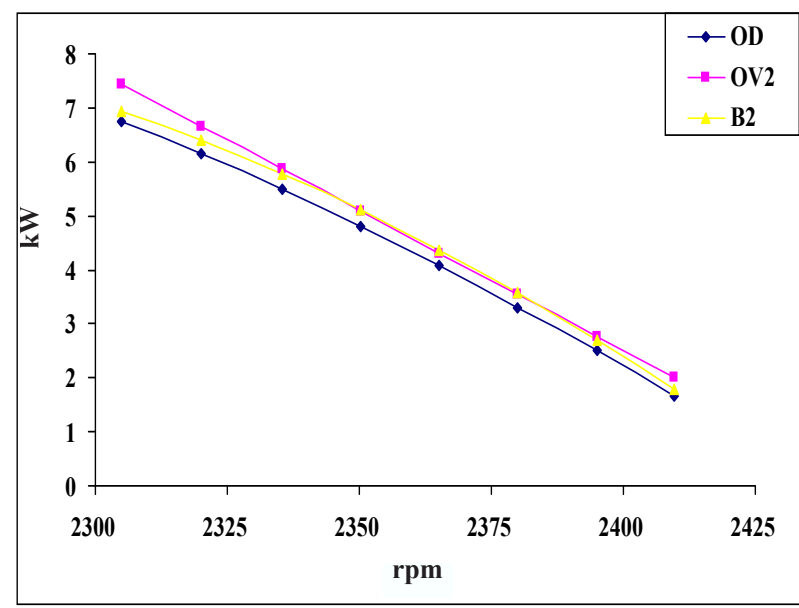

Figura 2. Curvas de potência do motor, estimadas a partir das equações apresentadas no Quadro 1

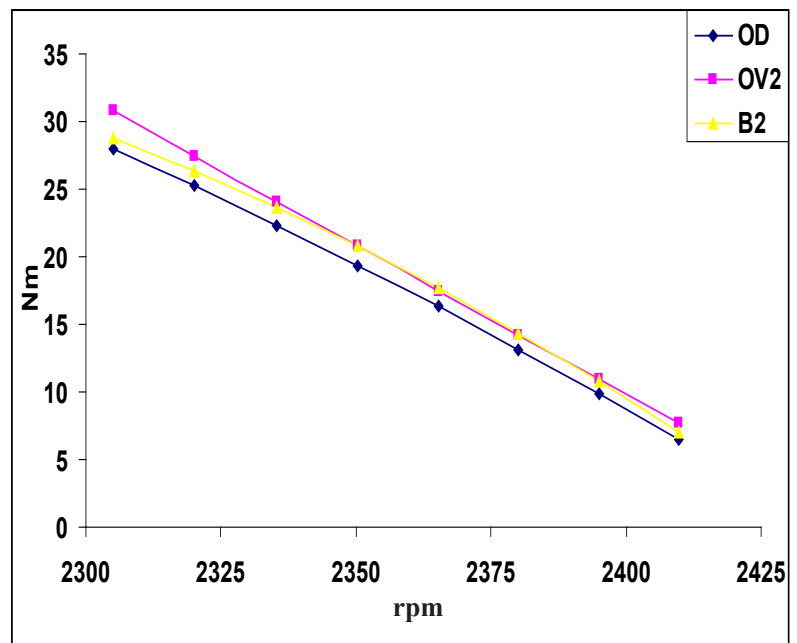

Figura 3. Curvas de torque do motor, estimadas a partir das equações apresentadas no Quadro 2

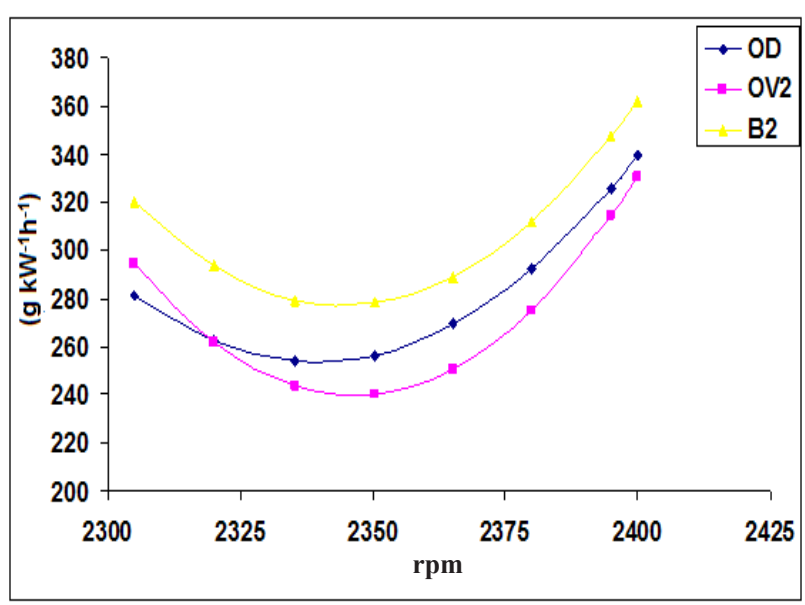

Figura 4. Curvas de consumo específico do motor, estimadas a partir das equações apresentadas no Quadro 3

\section{Rendimento do Motor com ORS}

Foram obtidas as equações apresentadas no Quadro 4, para as estimativas de potência, torque e consumo específico de combustível, para os diferentes níveis de combustíveis avaliados.

No Quadro 5 são apresentadas as equações gerais para estimar os valores de potência, torque e consumo específico, para qualquer dos combustíveis avaliados. Nestas equações, pode-se verificar que no caso do consumo específico, os níveis de ORS nos combustíveis não foram significativos a $5 \%$ pelo teste $t$, portanto, os valores do consumo específico podem ser considerados estatisticamente iguais para os combustíveis avaliados.

A potência e o torque do motor avaliado tiveram uma tendência a ter um melhor desempenho com o aumento da proporção de ORS no combustível, como pode ser observado na Figura 5 e 6.

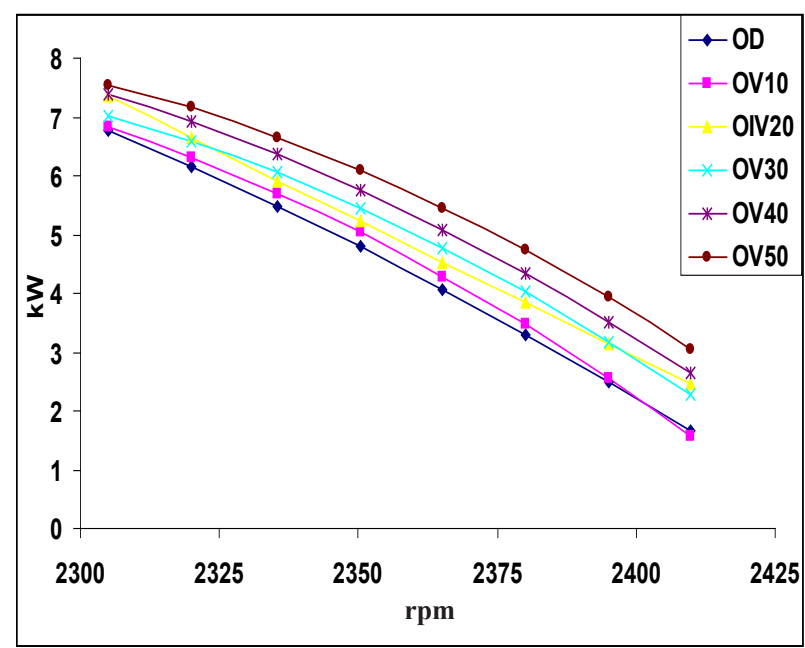

Figura 5. Curvas de Potência do Motor, segundo as equações de cada combustível, apresentadas na Quadro 4

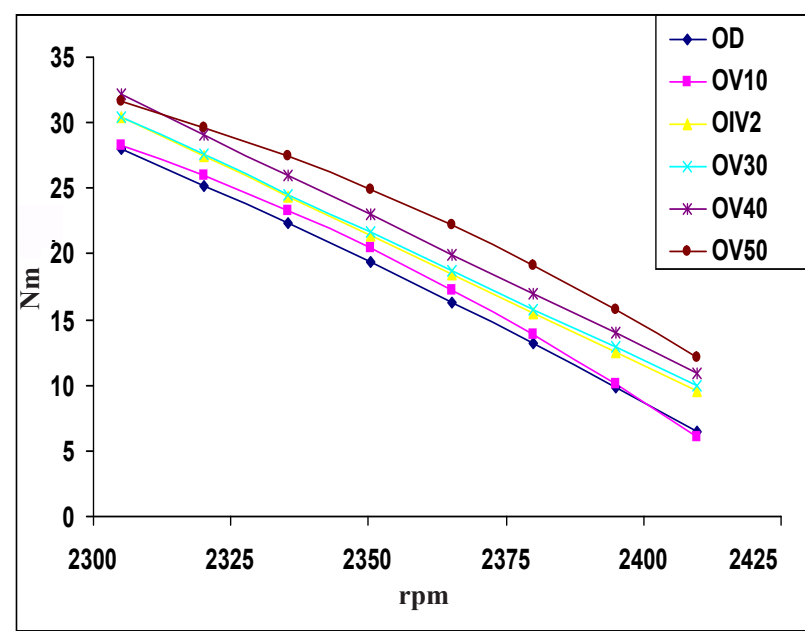

Figura 6. Curvas de torque do Motor, segundo as equações de cada combustível, apresentadas na Quadro 4 
O motor demonstrou uma tendência de aumento da potência proporcional à adição de ORS na mistura com OD. Para a condição de trabalho, na rotação de $2350 \mathrm{rpm}$, as potência sofreu um acréscimo de 5,15; 9,$11 ; 13,79 ; 20,08$ e $27,34 \%$, respectivamente para $\mathrm{OV}_{10}, \mathrm{OV}_{20}, \mathrm{OV}_{30}, \mathrm{OV}_{40}$ e $\mathrm{OV}_{50}$, conforme Figura 7.

Resultado semelhante foi encontrado por Maronhas (2008) na utilização de misturas de óleo de soja reutilizado com óleo diesel em um motor estacionário onde verificou-se que a potência e o torque do motor se apresentaram maiores com o acréscimo de óleo de soja reutilizado ao óleo Diesel.

No caso do torque o mesmo efeito foi notado, onde ocorreram acréscimo de torque na ordem de 5,46; 10,56; 11,$73 ; 18,65$ e $28.82 \%$, respectivamente para $\mathrm{OV}_{10}, \mathrm{OV}_{20}$ $\mathrm{OV}_{30} \mathrm{OV}_{40}$ e $\mathrm{OV}_{50}$.

A pesquisa de Siqueira (2010) na utilização de misturas de óleo de soja reutilizado com óleo diesel em um trator agrícola de pneus observou que com o acréscimo de OSR proporcionou um incremento no torque do motor.

Machado (2003) avaliando o desempenho do óleo de soja como combustível para motores diesel constatou que a mistura de 10 a 30\% de óleo de soja com óleo diesel proporcionou melhores resultados com relação as demais misturas com o maior índice de óleo vegetal.

Para o consumo específico de combustível observa uma tendência quando em rotações abaixo $2300 \mathrm{rpm}$ o motor tem consumo específico maior para as misturas do óleo de soja (ORS) e diesel, já para rotações a cima $2375 \mathrm{rpm}$, as misturas de ORS demonstraram um menor consumo em comparação com o diesel puro. As diferenças encontradas foram de $9,50,6,23,2,61,7,50$ e $9,26 \%$, respectivamente para OV10, OV20 OV30 OV40 e OV50. Na condição entre $2350 \mathrm{rpm}$ e $2375 \mathrm{rpm}$ observou-se o menor consumo de combustível. Siqueira (2010) observou que a mudança de combustível provocou variação no consumo especifico do motor de um trator agrícola de pneus, sendo que a mistura de $0 \%$ OD com $100 \%$ OSR obteve, maior consumo especifico comparada com as demais.

Inoue (2008) avaliando a utilização de óleo degomado de soja em motor de ciclo Diesel constatou que o consumo específico sofreu influência, aumentando com a adição de ODS no combustível, chegando a 8\% na condição OV100.

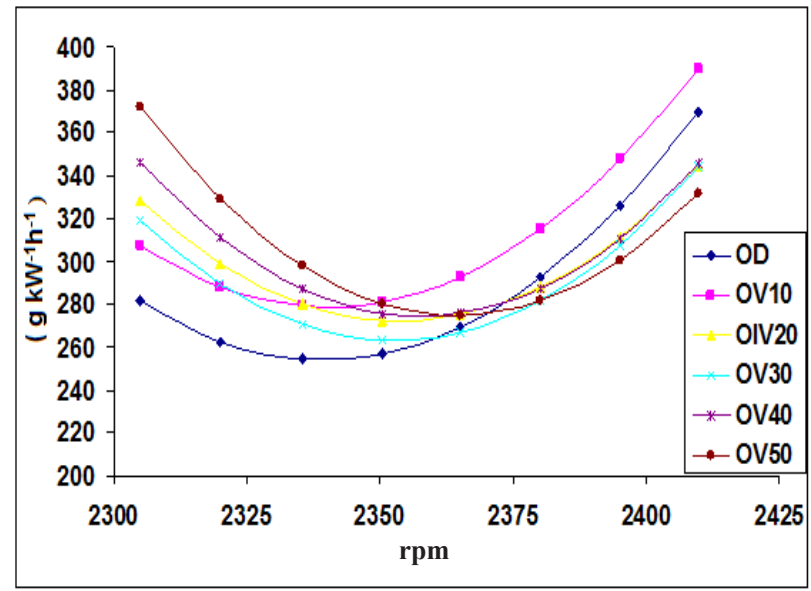

Figura 7.Curvas consumo específico de combustível pelo motor.

\section{Custo da utilização do ORS em substituição do óleo diesel.}

$\mathrm{Na}$ rotação de trabalho do motor a 2350rpm, como apresentado no Quadro 6, foram utilizados os valores da potência e do consumo específico, o custo de aquisição do ORS e do OD e a densidade dos combustíveis, para realizar o cálculo do custo com o combustível horário do motor. Em função da potência e do custo horário do combustível foi calculado o custo em $\mathrm{R} \$ \mathrm{~kW}^{-1} \mathrm{~h}^{-1}$.

Pode-se verificar no Quadro 6 que, mesmo ocorrendo consumo maior que o OD em nos outros combustíveis testados, o custo em $\mathrm{R} \$ \mathrm{~kW}^{-1} \mathrm{~h}^{-1}$ varia de forma pequena, devido o aumento da potência

Quadro 6. Analise do custo - $\mathrm{R} \$ \mathrm{~kW}^{-1} \mathrm{~h}^{-1}$

\begin{tabular}{cccccc}
\hline & $\begin{array}{c}\text { Consumo } \\
\text { Específico } \\
\left(\mathrm{g} / \mathrm{kW}^{-1} \mathrm{~h}^{-1}\right)\end{array}$ & $\begin{array}{c}\text { Potência } \\
(\mathrm{kW})\end{array}$ & $\begin{array}{c}\text { Custo } \\
\left(\mathrm{R} \$ \mathrm{~L}^{-1}\right)\end{array}$ & $\begin{array}{c}\text { Custo } \\
\left(\mathrm{R} \$ \mathrm{~h}^{-1}\right)\end{array}$ & $\begin{array}{c}\text { Custo } \\
\left(\mathrm{R} \$ \mathrm{~kW}^{-1} \mathrm{~h}^{-1}\right)\end{array}$ \\
\hline OD & 257 & 4,79 & 1,90 & 1,99 & 0,42 \\
\hline OV10 & 281 & 5,04 & 1,89 & 2,28 & 0,45 \\
\hline OV20 & 273 & 5,23 & 1,88 & 2,29 & 0,44 \\
\hline OV30 & 263 & 5,45 & 1,87 & 2,29 & 0,42 \\
\hline OV40 & 276 & 5,75 & 1,86 & 2,52 & 0,44 \\
\hline OV50 & 280 & 6,10 & 1,85 & 2,69 & 0,44 \\
\hline
\end{tabular}


do motor nos outros combustíveis testados. Assim, o custo de funcionamento do motor para a potência e rotação de trabalho será insignificante, ou seja, podendo ser utilizado qualquer um dos combustíveis.

Deve se levar em consideração que o ORS foi adquirido em supermercado, sendo assim, acreditase que se o mesmo fosse adquirido no atacado ou diretamente em cooperativas, este custo seria reduzido significativamente.

\section{CONCLUSÕES}

Pode-se concluir de acordo com os resultados obtidos neste trabalho que:

- O aquecimento do combustível é uma técnica viável para a redução da viscosidade;

- Ocorreu aumento de potência e torque em função do aumento da proporção de ORS;

- Em termos de consumo do motor, houve um aumento em função do aumento da

- proporção de ORS;

- $\mathrm{Na}$ análise econômica, se o ORS for adquirido em condições de atacado, pode se tornar viável;

- Outros trabalhos deverão ser realizados para avaliar o motor em condições de trabalho

\section{REFERÊNCIAS BIBLIOGRÁFICAS}

AGÊNCIA NACIONAL DO PETRÓLEO-ANP (2001). Portaria ANP No 310, de 27 de Dezembro de 2001. Diario Oficial da União de 28/12/2001

\section{ASSOCIAÇÃO BRASILEIRA DE NORMAS} TÉCNICAS. NBR 10441 Produtos de petróleo Líquidos transparentes e opacos - Determinação da viscosidade cinemática e cálculo da viscosidade dinâmica. Rio de Janeiro, 2007. 19p.

ASSOCIAÇÃO BRASILEIRA DE NORMAS TÉCNICAS. NBR 5484: motores alternativos de combustão interna de ignição por compressão (Diesel) ou ignição por centelha (Otto) de velocidade angular variável - ensaio - Método de ensaio. Rio de Janeiro, 1985. 8p.

ASSOCIAÇÃO BRASILEIRA DE NORMAS TÉCNICAS. NBR 1585: Veiculos rodoviários - códigos de ensaios de motores - potencia liquida. Rio de Janeiro, 1996. 26p.

BARGUER, E.L., LILJEDAHL, J.B., CARLETON, W.M., MCKIBBEN, E.G. Tratores e seus motores. São Paulo: Edgar Blucher, 1966. 398p.

CASTRO, A.A. Extração, caracterização físicoquimica, nutricional e reológica do azeite do coco babaçu (Orbignya spp). 1999. 65 f. Dissertação (Mestrado em Engenharia Agrícola) - Departamento de Engenharia Agrícola, Universidade Federal da Paraíba, Campina Grande.

DELGADO, L.M. Los cultivos no alimentarios como alternativa al abandono de tierras. Madrid, España: Agrícola Española, 1994. 127p.

FENDER, T.R. Por que usar puro óleo vegetal como combustível?. Via Jus, Porto Alegre, RS. Disponível em:http://www.viajus. com.br/viajus.php?pagina $=$ artigos\&id=16 Acesso em: 22 jul. 2009

GONZÁLEZ, J.F. Cultivos energéticos para la Península Ibérica. In: DELGADO, L.M. Los cultivos no alimentarios como alternativa al abandono de tierras. Madrid, España: Agricola Española, 1994. p.27-41.

IICA. Informe Sobre a Situação e Perspectivas da Agroenergia e dos Biocombustíveis no Brasil. 44p. 2007

INOUE, G.H.;VIEIRA, L. B. ; SANTOS, G. L.; RESENDE, R. C.; FILJO, A. F. L. Nota técnica: avaliação do desgaste de um motor de ciclo diesel alimentado com misturas de óleo vegetal e diesel. Engenharia na Agricultura, v.17, n.4, p.294-302, 2009.

INOUE, G.H. Utilização de óleo vegetal em motor estacioná rio de ciclo diesel. Viçosa, MG: UFV, 2008. $102 \mathrm{f}$. Tese (Doutorado em Engenharia Agrícola) - Universidade Federal de Viçosa, Viçosa.

MINISTÉRIO DE MINAS E ENERGIA - MME, Balanço Energético Nacional. Análise Energética Brasileira - período 1970 a 2002. Brasília. 2004a. Disponível em www.mme.gov.br. Acesso em: 05/04/2004. 
MACHADO, P.R.M; Avaliação de desempenho do óleo de soja como combustível para motores diesel. Santa Maria, RS: UFSM, 2003. 212f. Dissertação (Mestrado em Engenharia Agrícola)Universidade Federal de Santa Maria. Centro de Ciências Rurais, Santa Maria.

MARONHAS, M.E.S. Utilização de Misturas de Óleo de Soja Reutilizado com Óleo Diesel em um Motor Estacionário. Viçosa, MG: UFV, 2008. 18 f. Relatório Final PIBIC/CNPq (Bolsista PIBIC/CNPq) - Universidade Federal de Viçosa, Viçosa.
MORAES, J.R. Manual dos óleos vegetais e suas possibilidades energéticas. CNI. 1980. 78p.

SANT'ANNA, J.P. Biodiesel alimenta motor da economia. Revista Química e Derivados, v. 414, abril de 2003. www.quimica.com.br. Acesso em: 29/04/2004.

SIQUEIRA, C.W; Desempenho de um trator agrícola de pneus funcionando com misturas de óleo diesel e óleo de soja reutilizado. Viçosa, MG: UFV, 2010. 71f. Dissertação (Mestrado em Engenharia Agricola) - 